\title{
EFEITO RESIDUAL DO TARO CONSORCIADO COM LEGUMINOSAS NOS TEORES DOS PIGMENTOS FOTOSSINTETIZANTES DE BRASSICA OLERACEA
}

\author{
Rafael Costa de Sant'Ana' ${ }^{1}$ \\ Hediberto Nei Matiello ${ }^{2}$ \\ Alessandra do Nascimento Pedro ${ }^{3}$ \\ Stanley Bravo Buffon ${ }^{4}$ \\ Dayane Littig Barker ${ }^{5}$ \\ Sara Dousseau Arantes ${ }^{6}$ \\ Rafael Zucateli da Vitoria ${ }^{7}$ \\ Felipe de Tássio Gonçalves de Oliveira ${ }^{8}$ \\ Renan Garcia Malikouski ${ }^{9}$ \\ Felipe Pereira Dal'Co ${ }^{10}$ \\ João Nacir Colombo'11
}

Resumo: Avaliou-se o efeito residual do consórcio taro e crotalarias sob duas posições de plantio nos teores dos pigmentos fotossintetizantes do repolho (Brassica oleracea var. capitata L.). O experimento foi conduzido no Instituto Federal do Espírito Santo - campus Santa Teresa no período de agosto a novembro de 2015, dois meses após a colheita do cultivo anterior. O delineamento experimental utilizado foi o de blocos casualizados, em esquema fatorial com dois fatores para posição (distribuição espacial nos sentidos norte-sul e leste-oeste) e três fatores para a cultura (efeito residual do consórcio taro (Colocassia esculenta (L.) Schott) com a Crotalaria juncea, Crotalaria spectabilis, mais o taro solteiro) correspondendo a 6 tratamentos com 4 repetições. Não foram observadas diferenças estatísticas entre os teores de pigmentos fotossintetizantes para os fatores consórcio e posição, provavelmente este resultado foi devido ao elevado vigor do hibrido utilizado, ASTRUS PLUS.

Palavras-chave: Crotalaria juncea; Crotalaria spectabilis; Colocasia esculenta; Brassica oleracea var.capitata.

\footnotetext{
1 Instituto Capixaba de Pesquisa, Assistência Técnica e Extensão Rural - INCAPER, Centro Regional de Desenvolvimento Rural -Centro Norte, Laboratório de Fisiologia Vegetal e Pós-Colheita, Linhares, ES, Brasil. Email: santanaagronomia@gmail.com.

${ }_{2}$ Instituto Federal do Espírito Santo - IFES, Campus Santa Teresa, Santa Teresa, ES, Brasil. E-mail: hedibertonm@ifes.edu.br.

3 Instituto Federal do Espírito Santo - IFES, Campus Santa Teresa, Santa Teresa, ES, Brasil. E-mail: alessandra14np@gmail.com

${ }^{4}$ Instituto Capixaba de Pesquisa, Assistência Técnica e Extensão Rural - INCAPER, Centro Regional de Desenvolvimento Rural -Centro Norte, Laboratório de Fisiologia Vegetal e Pós-Colheita, Linhares, ES, Brasil. Email: acordatrevo@hotmail.com.

${ }^{5}$ Universidade Federal do Espírito Santo - UFES, Centro Universitário Norte do Espírito Santo - CEUNES, São Mateus, ES, Brasil. E-mail: dayanelittig@hotmail.com.

${ }^{6}$ Instituto Capixaba de Pesquisa, Assistência Técnica e Extensão Rural - INCAPER, Centro Regional de Desenvolvimento Rural -Centro Norte, Laboratório de Fisiologia Vegetal e Pós-Colheita, Linhares, ES, Brasil. Email: saradousseau@yahoo.com.br.

7 Universidade Federal do Espírito Santo - UFES, Centro Universitário Norte do Espírito Santo - CEUNES, São Mateus, ES, Brasil. E-mail: zucateli_rafael@hotmail.com.

8 Instituto Capixaba de Pesquisa, Assistência Técnica e Extensão Rural - INCAPER, Centro Regional de Desenvolvimento Rural -Centro Norte, Laboratório de Fisiologia Vegetal e Pós-Colheita, Linhares, ES, Brasil. Email: felipe_dtassio@hotmail.com.

9 Instituto Federal do Espírito Santo - IFES, Campus Santa Teresa, Santa Teresa, ES, Brasil. E-mail: renan_malikouski@hotmail.com.

10 Instituto Federal do Espírito Santo - IFES, Campus Santa Teresa, Santa Teresa, ES, Brasil. E-mail: felipepdalcol@gmail.com.

11 Instituto Federal do Espírito Santo - IFES, Campus Santa Teresa, Santa Teresa, ES, Brasil. E-mail: joaonacirc@yahoo.com.br.
} 